OPEN ACCESS

Fabrication of a Micro-Needle Sensor Based on Copper Microspheres and Polyaniline Film for Nitrate Determination in Coastal River Waters

To cite this article: Ying Li et al 2019 J. Electrochem. Soc. 166 B1038

View the article online for updates and enhancements. 


\title{
(ESS) \\ Fabrication of a Micro-Needle Sensor Based on Copper Microspheres and Polyaniline Film for Nitrate Determination in Coastal River Waters
}

\author{
Ying Li, ${ }^{1,2}$ Haitao Han, ${ }^{2,3,4, z}$ Dawei Pan, ${ }^{2,3,4}$ and Peiqing Zhang ${ }^{1, z}$ \\ ${ }^{I}$ School of Chemistry and Chemical Engineering, Yantai University, Yantai 264005, People's Republic of China \\ ${ }^{2}$ CAS Key Laboratory of Coastal Environmental Processes and Ecological Remediation, Research Center for Coastal \\ Environment Engineering Technology of Shandong Province, Yantai Institute of Coastal Zone Research, Chinese \\ Academy of Science, Yantai 264003, People's Republic of China \\ ${ }^{3}$ Center for Ocean Mega-Science, Chinese Academy of Sciences, Qingdao 266071, People's Republic of China \\ ${ }^{4}$ University of Chinese Academy of Sciences, Beijing 100049, People's Republic of China
}

\begin{abstract}
In this work, a new micro-needle sensor based on copper microspheres (CuMSs) and polyaniline (PANI) film was fabricated through electrodeposition method and used for the voltammetric determination of nitrate $\left(\mathrm{NO}_{3}^{-}\right)$in coastal river samples. The PANI film functions as the conductive polymer and adhesion agent to immobilize the CuMSs which has good electrocatalytic property for the reduction of $\mathrm{NO}_{3}^{-}$. The CuMSs could be more easily deposited on the micro-needle electrode (MNE) surface with PANI film as adhesion agent. The physical and electrochemical properties of the as-prepared micro-needle electrode were characterized by different techniques. CuMSs with diameter of about 0.5 to $1 \mu \mathrm{m}$ were decorated on the PANI film modified micro-needle electrode. The unique structure of micro-needle electrode and excellent properties of CuMSs and PANI film make the micro-needle sensor possesses the advantages of larger specific surface and high electrocatalytic property towards the reduction of $\mathrm{NO}_{3}^{-}$. The micro-needle electrode based on CuMSs and PANI film shows a linear response to $\mathrm{NO}_{3}^{-}$in the concentrations ranging from 0.02 to $6 \mathrm{mM}\left(\mathrm{R}^{2}=\right.$ $0.995)$ with the detection limit of $8 \mu \mathrm{M}$. More importantly, the determination of $\mathrm{NO}_{3}^{-}$in coastal river water samples was achieved with the micro-needle electrode with satisfactory results.

(C) The Author(s) 2019. Published by ECS. This is an open access article distributed under the terms of the Creative Commons Attribution 4.0 License (CC BY, http://creativecommons.org/licenses/by/4.0/), which permits unrestricted reuse of the work in any medium, provided the original work is properly cited. [DOI: 10.1149/2.1281912jes]

(cc) BY
\end{abstract}

Manuscript submitted March 29, 2019; revised manuscript received July 19, 2019. Published August 2, 2019.

Nitrogen which plays a crucial role in many biogeochemical cycles is an essential component for marine phytoplankton growth. ${ }^{1}$ Nitrate $\left(\mathrm{NO}_{3}^{-}\right)$is the most stable substance in various forms of nitrogenous compounds under aerobic conditions. It is the principal form of reactive inorganic nitrogen in seawater and therefore a major nutrient to support primary productivity in the surface ocean. ${ }^{2}$ It is also the final decomposition product of nitrogenous organic matter through inorganication. In natural water, $\mathrm{NO}_{3}^{-}$as well as nitrite and ammonium, are the major forms of dissolved inorganic nitrogen (DIN) that is assimilated by phytoplankton for their growth. ${ }^{3-5}$ Considering the spatial and temporal distribution of $\mathrm{NO}_{3}^{-}$concentrations in the ocean are affected by both physical and biogeochemical processes, accurate determination of $\mathrm{NO}_{3}^{-}$concentrations can provide essential information for understanding such processes. Accurate quantification of $\mathrm{NO}_{3}^{-}$is essential for understanding the oceanic nitrogen cycle and the dynamics of marine ecosystems. The common methods for detecting $\mathrm{NO}_{3}^{-}$include chemiluminescence, fluorescence, chromatography, and ion chromatography. However, these methods are relatively time-consuming, fiddly and costly. ${ }^{6}$ When compared with other methods, electrochemical method has the advantages of time saving, low cost, inherent miniaturization and portability, which make it interesting in the field of analytical chemistry. ${ }^{7}$ Some traditional electrodes like glassy carbon electrode, screen-printed electrode, and bismuth bulk electrode are commonly used as the working electrode for $\mathrm{NO}_{3}^{-}$determination. ${ }^{8-10}$ However, all the electrodes need polishing process before electrochemical experiments which are relatively time-consuming. What's more, they can't satisfy the need of sensitivity for detecting $\mathrm{NO}_{3}^{-}$in coastal river water. So it is of great necessity to fabricate a novel, sensitive and easy-used electrode to achieve the determination of $\mathrm{NO}_{3}^{-}$in coastal river water samples.

Acupuncture is a traditional medical technology originating from China. There are numerous kinds of materials for acupuncture needles on the market, such as gold, silver, copper, and stainless steel. ${ }^{11-13}$ Among them, stainless steel is most widely used due to its flexi-

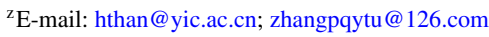

bility and low price. ${ }^{14}$ It has been reported that the stainless steel acupuncture needle can be manufactured as micro-needle electrode (MNE) for electrochemical and bio-sensing applications. ${ }^{15-18}$ It exhibits much superior properties in sensitivity and detection limit than the conventional electrode because of its unique shape and size. Besides, there is no polishing process needed before the electrochemical experiments.

Lots of functional materials such as copper $(\mathrm{Cu})$, nickel, copper-nickel alloys, copper-palladium alloys, platinum, lead, silver, rhodium, boron-doped diamond, gold etc. have been modified on bare electrodes to enhance their electrochemical properties. As for $\mathrm{NO}_{3}^{-}$ detection, copper material has been fully used. ${ }^{19-21}$ Recently, scientists have made many efforts directing towards the synthesis of $\mathrm{Cu}$ micro-nanostructures, such as nanoparticles, nanoplates, nanoflowers, nanorods/wires, nanoribbons, micro-dandelions, hollow spheres, spindles, nanofilms, and hierarchical nanostructures, and many of them were examined for sensing applications. ${ }^{22-27}$ As to our knowledge, copper microspheres (CuMSs) modified micro-needle electrode for $\mathrm{NO}_{3}^{-}$detection has ever been reported previously. However, considering the smooth surface of stainless steel needle, CuMSs are not easy to be modified on the bare MNE. To solve this problem, some adhesion agents should be adopted to conduct the surface modification of MNE.

As a kind of conducting polymer with excellent conductivity, good environmental and chemical stability, Polyaniline (PANI) has been applied to many fields, such as sensing, energy storage, anticorrosion and adsorption. ${ }^{28-33}$ And nanomaterials such as $\mathrm{Ag}, \mathrm{SiO}_{2}, \mathrm{TiO}_{2}, \mathrm{Si}$ and $\mathrm{Cu}$ have ever been reported to combine with PANI due to its abundant binding sites. Moreover, the abundant $-\mathrm{NH}_{2}$ group on the PANI chains can enhance the bonding ability with metal nanomaterials. ${ }^{34-37}$ So, it is expected that the PANI film could be deposited on the MNE firstly to make the CuMSs deposited more easily and stably which has good electrocatalytic property for the reduction of $\mathrm{NO}_{3}^{-}$.

In this work, PANI and CuMSs modified MNE (CuMSs/PANI/MNE) has been fabricated for the determination of $\mathrm{NO}_{3}^{-}$in coastal river samples by DPV. PANI film as the conductive polymer and adhesion agent offers a lot of binding sites to combine with CuMSs. CuMSs were employed as the excellent catalytic 
material for the electrochemical reduction of $\mathrm{NO}_{3}^{-}$. What's more, the PANI/CuMSs/MNE exhibited high sensitivity, selectivity, and stability for the voltammetric detection of $\mathrm{NO}_{3}^{-}$in coastal river water samples.

\section{Experimental}

Materials and reagents.-Copper sulfate $\left(\mathrm{CuSO}_{4}\right)$, sulfuric acid $\left(\mathrm{H}_{2} \mathrm{SO}_{4}\right)$, sodium sulfate $\left(\mathrm{Na}_{2} \mathrm{SO}_{4}\right)$, sodium nitrate $\left(\mathrm{NaNO}_{3}\right)$, enthanol $\left(\mathrm{CH}_{3} \mathrm{CH}_{2} \mathrm{OH}\right)$ and aniline was purchased from Sinopharm Chemical Reagent Co. Ltd. (Shanghai, China). The supporting electrolyte $(\mathrm{pH}$ 2) in all experiments was $0.1 \mathrm{M} \mathrm{Na}_{2} \mathrm{SO}_{4}$ solution containing $0.5 \mathrm{M}$ $\mathrm{H}_{2} \mathrm{SO}_{4}$. Deionized water obtained from Pall Cascada laboratory water system was used throughout. All chemicals were analytical reagents and used without further purification. $0.1 \mathrm{M}$ aniline solution was prepared in $0.5 \mathrm{M} \mathrm{H}_{2} \mathrm{SO}_{4}$ for electrodeposition. Stainless steel acupuncture needles were purchased from Suzhou Medical Appliance Factory Co. Ltd. (Suzhou, China). Silicone rubber was purchased from Liyang Kangda Chemical Co. Ltd., China.

Apparatus.-Electrochemical experiments including cyclic voltammetry $(\mathrm{CV})$, chronoamperometry, differential pulse voltammetry (DPV), and electrochemical impedance spectroscopy (EIS) were performed on a CHI660E electrochemical workstation (ChenHua, Shanghai, China). The morphologies of the bare and modified electrodes were characterized by scanning electron microscopy (SEM, Hitachi S-4800 microscope, Japan) and Energy dispersive X-ray spectroscopy (EDS, Hitachi S-4800 microscope, Japan). The modified MNE served as the working electrode, with $\mathrm{Ag} / \mathrm{AgCl}$ (3.0 $\mathrm{M} \mathrm{KCl}$ ) and platinum foil serving as the reference and counter electrode, respectively. All potentials were measured with respect to the $\mathrm{Ag} / \mathrm{AgCl}$ reference electrode.

Preparation of the CuMSs/PANI/MNE.-Firstly, the MNE was manufactured by stainless steel acupuncture needles and the manufacture procedure was similar to our previous work with modification. ${ }^{17}$ The needle was sealed with silicone rubber with needle tip about 1-2 $\mathrm{mm}$ and needle handle out for the sensing surface and electrode connection, respectively. Secondly, the PANI film modified MNE (PANI/MNE) was prepared by cycling the potential between -0.3 to $+0.9 \mathrm{~V}$ at a scan rate of $50 \mathrm{mV} / \mathrm{s}$ for 10 circles in $0.5 \mathrm{M} \mathrm{H}_{2} \mathrm{SO}_{4}$ solution with $0.1 \mathrm{M}$ aniline. After the electro-polymerization process, $\mathrm{CV}$ was conducted to verify the successful modification of PANI film. Then the modification of CuMSs on PANI/MNE surface was achieved by electrodepositing in $0.5 \mathrm{M} \mathrm{CuSO}_{4}$ solution at the potential of $-0.25 \mathrm{~V}$ for $12 \mathrm{~s}$. And the CuMSs/PANI/MNE was obtained eventually after the electrode was cleaned carefully and dried at room temperature.

Electrochemical analysis procedure.-The CV plots were obtained in $0.1 \mathrm{M} \mathrm{Na}_{2} \mathrm{SO}_{4}(\mathrm{pH} 2)$ from -0.80 to $-0.30 \mathrm{~V}$. The EIS of different electrodes was conducted in $5 \mathrm{mM} \mathrm{K}_{3}\left[\mathrm{Fe}(\mathrm{CN})_{6}\right] / \mathrm{KCl}$ $(0.5 \mathrm{M})$ solution. Before the conduction of EIS experiments, the open circuit potentials were measured firstly, and set as $0.19 \mathrm{~V}$ for MNE, $0.24 \mathrm{~V}$ for PANI/MNE, and $0.23 \mathrm{~V}$ for CuMSs/PANI/MNE, respectively. The current responses of $\mathrm{NO}_{3}^{-}$on different MNEs were investigated by DPV using the following parameters: initial potential of $-0.30 \mathrm{~V}$, final potential of $-0.80 \mathrm{~V}$, amplitude of $0.05 \mathrm{~V}$, potential incremental of $0.004 \mathrm{~V}$, pulse width of $0.05 \mathrm{~s}$, pulse period of $0.5 \mathrm{~s}$ and quiet time of $2 \mathrm{~s}$.

\section{Results and Discussion}

Characterization of the CUMSs/PANI/MNE.-The morphologies and elemental composition of the modified electrodes were characterized by SEM and EDS, respectively. SEM images of MNE (a, b), PANI/MNE (c), and CuMSs/PANI/MNE (d) are shown in Fig. 1. It can be seen from Figs. 1a and 1b that the surface of MNE is very smooth and the needle tip diameter is around $5 \mu \mathrm{m}$ (as shown in

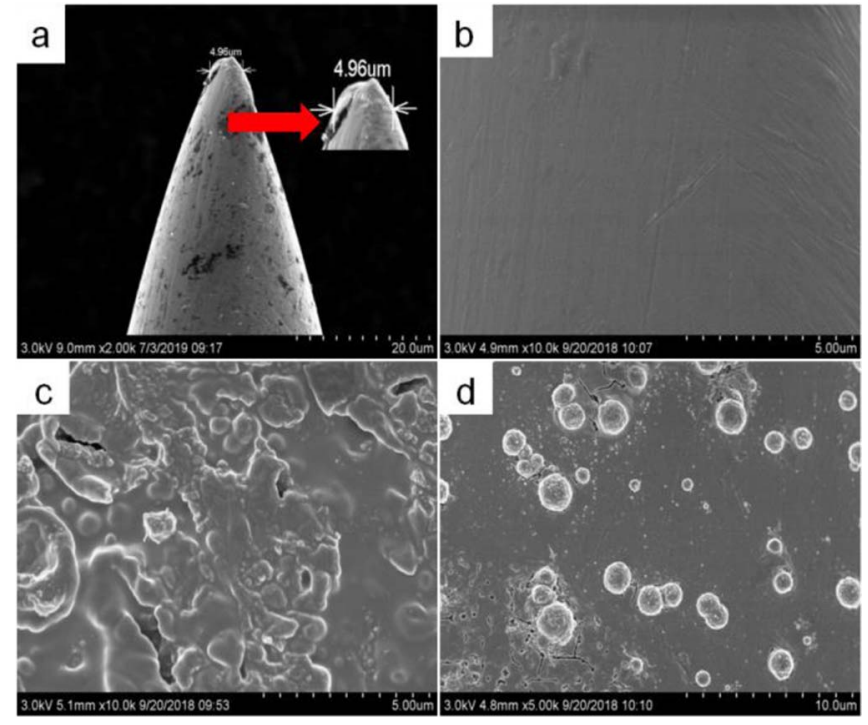

Figure 1. Typical SEM images of MNE (a, b), PANI/MNE (c), and CuMSs/PANI/MNE (d). Insert shows the amplified and size-marked needle tip of MNE (a).

the insert in Fig. 1a). After the modification of PANI, it can be seen clearly from Fig. 1c that there is a film structure covered on the surface of MNE. Fig. 1d shows that CuMSs with the diameter about 0.5 to $1 \mu \mathrm{m}$ are distributed on the PANI film surface after the process of electrochemical deposition. And according to the Randles-Sevcik equation, ${ }^{38-40}$ the electrochemical areas of bare MNE, PANI/MNE and $\mathrm{CuMSs} / \mathrm{PANI} / \mathrm{MNE}$ were calculated as $1.75 \times 10^{-6}, 1.04 \times 10^{-5}$, and $2.94 \times 10^{-5} \mathrm{~cm}^{2}$, respectively. The EDS pattern is shown in Fig. 2. It can be observed that $\mathrm{C}, \mathrm{N}, \mathrm{O}, \mathrm{Al}, \mathrm{Si}, \mathrm{Fe}, \mathrm{Ni}, \mathrm{Cr}$ are the major elements of the MNE (Fig. 2a). After the modification of PANI, the weight percentage of $\mathrm{C}$ and $\mathrm{N}$ increased (Fig. 2b). The insert in Fig. 2 shows the region in the SEM image where the EDS pattern of CuMSs/PANI/MNE (Fig. 2c) was obtained. Obviously, after the deposition process in $\mathrm{CuSO}_{4}$ solution, the presence of typical $\mathrm{Cu}$ peaks at about 0.1 and 8.1 $\mathrm{KeV}$ indicates the successful fabrication of CuMSs/PANI/MNE. The results of SEM and EDS characterization demonstrate that both PANI film and CuMSs have been successfully modified on the MNE. What's more, the excellent electrocatalytic property of CuMSs and adhesion

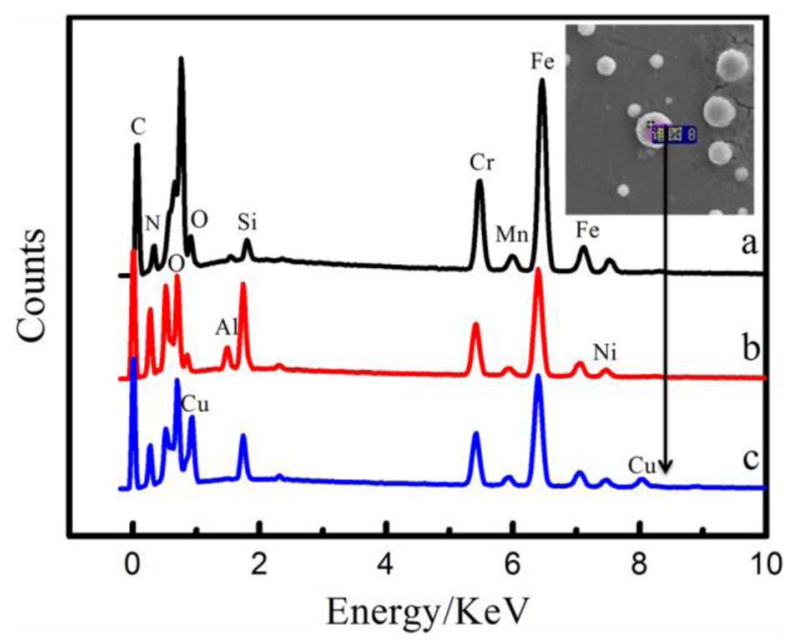

Figure 2. EDS spectrum of MNE (a), PANI/MNE (b), and CuMSs/PANI/MNE (c). Insert shows the region in the SEM image where the EDS pattern of CuMSs/PANI/MNE was obtained. 


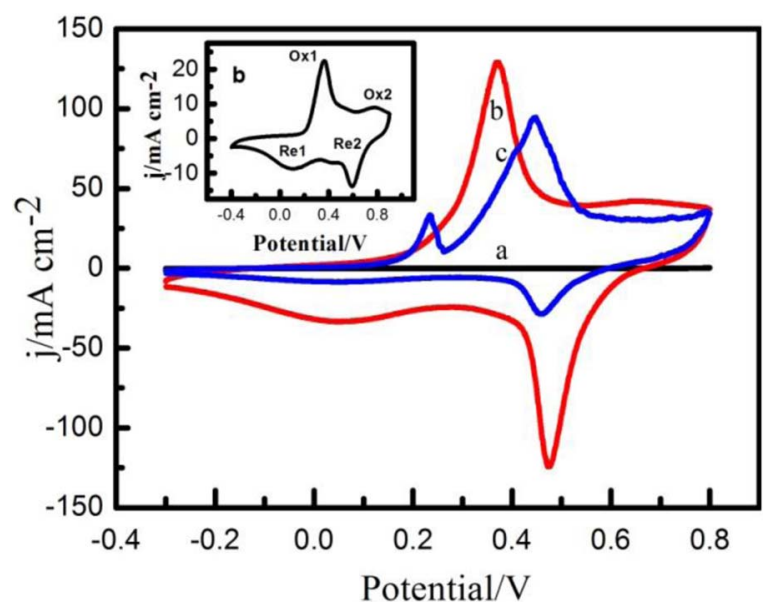

Figure 3. CV plots of the bare MNE (a), PANI/MNE (b), and CuMSs/PANI/MNE (c) in $0.1 \mathrm{M} \mathrm{Na}_{2} \mathrm{SO}_{4}$ (pH 2) solution. Insert shows the $\mathrm{CV}$ plot of PANI/MNE in $0.1 \mathrm{M} \mathrm{Na}_{2} \mathrm{SO}_{4}$ solution ( $\mathrm{pH}$ 2) from $-0.4-0.9 \mathrm{~V}$.

ability of PANI film will be helpful for the improvement of detection performance for $\mathrm{NO}_{3}^{-}$in sensitivity and stability respectively.

Electrocatalysis of the CuMSs/PANI/MNE.-The cyclic voltammograms of bare MNE (a), PANI/MNE (b), and CuMSs/PANI/MNE (c) are shown in Fig. 3. The electrochemical properties of CuMSs/ PANI/MNE were investigated by $\mathrm{CV}$ in $0.1 \mathrm{M} \mathrm{Na}_{2} \mathrm{SO}_{4}(\mathrm{pH} 2$ ) solution. There is no characteristic peak showed in the voltammogram of MNE. However, as can be seen from the CV plot of PANI/MNE showed in the insert of Fig. 3, there are two groups of redox peaks labelled as $\mathrm{Ox} 1(0.5 \mathrm{~V}), \operatorname{Re} 1(0.1 \mathrm{~V}), \mathrm{Ox} 2(0.8 \mathrm{~V})$, and $\operatorname{Re} 2(0.6$ $\mathrm{V})$, respectively. After the deposition of CuMSs on the surface of PANI/MNE, an additional oxidation peak at $0.18 \mathrm{~V}$ appears on the $\mathrm{CV}$ plot of CuMSs/PANI/MNE. Considering that the difference between $\mathrm{CuMSs} / \mathrm{PANI} / \mathrm{MNE}$ and PANI/MNE is the presence of CuMSs, the additional peak in Fig. $3 \mathrm{c}$ at $0.18 \mathrm{~V}$ was believed as the typical peak of CuMSs. ${ }^{41}$ Moreover, thanks to the fast electron transfer of CuMSs and PANI film, the background current density of the modified MNEs is relatively larger than that of bare MNE.

Electrochemical impedance spectrum (EIS) was used to verify the capability of electron transfer of different electrodes, as well as the combined effects of CuMSs and PANI. The semicircle diameter of EIS, equal to the electron transfer resistance (Ret), is depended on the electron transfer kinetics of the redox probe at the electrode surface. The nyquist diagrams of bare MNE (a), PANI/MNE (c), and CuMSs/PANI/MNE (b) were measured in $5 \mathrm{mM}$ $\mathrm{Fe}(\mathrm{CN})_{6}{ }^{3-} / \mathrm{Fe}(\mathrm{CN})_{6}{ }^{4-}$ solution containing $0.5 \mathrm{M} \mathrm{KCl}$ which shown in Fig. 4. By comparing the semicircle diameters of different electrodes, it can be concluded that the Ret of PANI/MNE is significantly smaller than that of MNE. This phenomenon indicates the excellent ability of PANI in facilitating electron transfer process of the $\left[\mathrm{Fe}(\mathrm{CN})_{6}\right]^{3-} /\left[\mathrm{Fe}(\mathrm{CN})_{6}\right]^{4-}$ redox process. However, the Ret becomes larger after the deposition of CuMSs on the PANI/MNE indicating the weaker electro-conductive ability of CuMSs than PANI film. Although it has a relatively weak electro-conductivity than PANI film, $\mathrm{CuMSs}$ show excellent electrocatalytic performance for the reduction of $\mathrm{NO}_{3}^{-}$(as discussed below). These results also demonstrate the successful fabrication of CuMSs/PANI/MNE.

It has been reported that $\mathrm{NO}_{3}{ }^{-}$reduction in sufficient acidic conditions can occur as an eight-electron process according to the following equation: ${ }^{41}$

$$
\mathrm{NO}_{3}^{-}+8 \mathrm{e}^{-}+10 \mathrm{H}^{+} \rightarrow 3 \mathrm{H}_{2} \mathrm{O}+\mathrm{NH}_{4}^{+}
$$

DPV was adopted to investigate the electrochemical reduction of $\mathrm{NO}_{3}^{-}$on CuMSs/PANI/MNE (insert in Fig. 5) and voltammetric responses of $\mathrm{NO}_{3}^{-}$on different MNEs (Fig. 5). As the insert plot in Fig. 5

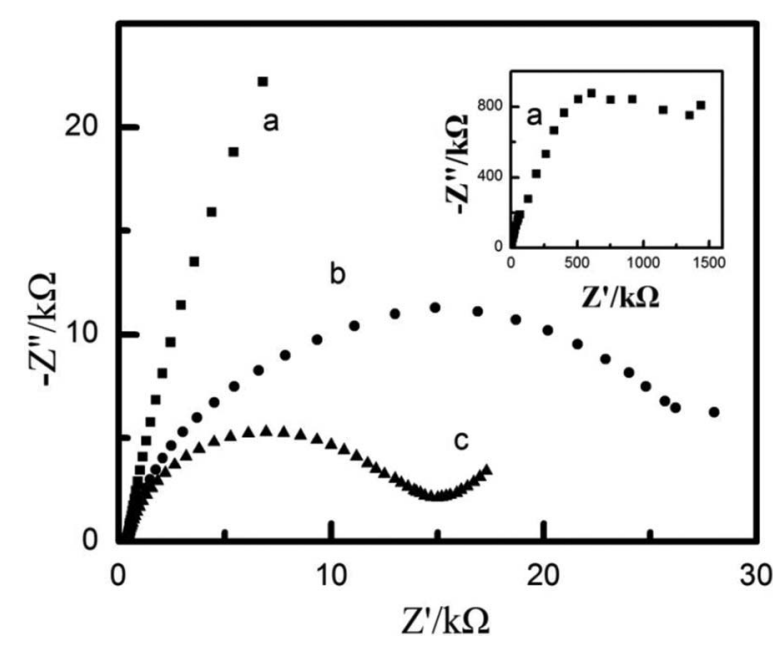

Figure 4. Nyquist plots of the bare MNE (a), CuMSs/PANI/MNE (b), and $\mathrm{PANI} / \mathrm{MNE}(\mathrm{c})$ in $0.5 \mathrm{M} \mathrm{KCl}$ solution containing $5 \mathrm{mM} \mathrm{K}_{3}\left[\mathrm{Fe}(\mathrm{CN})_{6}\right]$.

shown, there is no any peak on the DPV plot of CuMSs/PANI/MNE without $\mathrm{NO}_{3}^{-}$added. When $1 \mathrm{mM} \mathrm{NO}_{3}{ }^{-}$added into the electrolyte, a sharp reduction peak at about $-0.53 \mathrm{~V}$ can be observed. So, the reduction peak is caused by the electrochemical reduction of $\mathrm{NO}_{3}^{-}$. Additionally, it can be seen from the DPV responses of bare MNE (a), PANI/MNE (b), and CuMSs/PANI/MNE (c) in $0.1 \mathrm{M} \mathrm{Na}_{2} \mathrm{SO}_{4}$ solution ( $\mathrm{pH}$ 2) with $1 \mathrm{mM} \mathrm{NO}_{3}^{-}$that there is the reduction peak of $\mathrm{NO}_{3}^{-}$only on the CuMSs/PANI/MNE. There is no doubt that the CuMSs/PANI/MNE shows the best performance for $\mathrm{NO}_{3}^{-}$voltammetric detection thanks to the excellent catalytic performance of CuMSs. ${ }^{42}$ And the function of PANI film is to improve the combining ability between CuMSs and MNE which is helpful for the improvement of stability of the functional MNE.

Useful information involving electrochemical mechanism generally can be acquired from the relationship between current density and scan rate. Therefore, the voltammetric behavior of $\mathrm{CuMSs} / \mathrm{PANI} / \mathrm{MNE}$ at different scan rates was investigated in $0.1 \mathrm{M}$ $\mathrm{Na}_{2} \mathrm{SO}_{4}$ solution ( $\mathrm{pH}$ ) with $1 \mathrm{mM} \mathrm{NO}_{3}^{-}$. As shown in Fig. 6, the current density shows a linear relationship with the square root of scan rate in the range of 10 to $300 \mathrm{mV} / \mathrm{s}$. It is obvious that the reduction of $\mathrm{NO}_{3}^{-}$on CuMSs/PANI/MNE is a diffusion controlled process. ${ }^{43}$

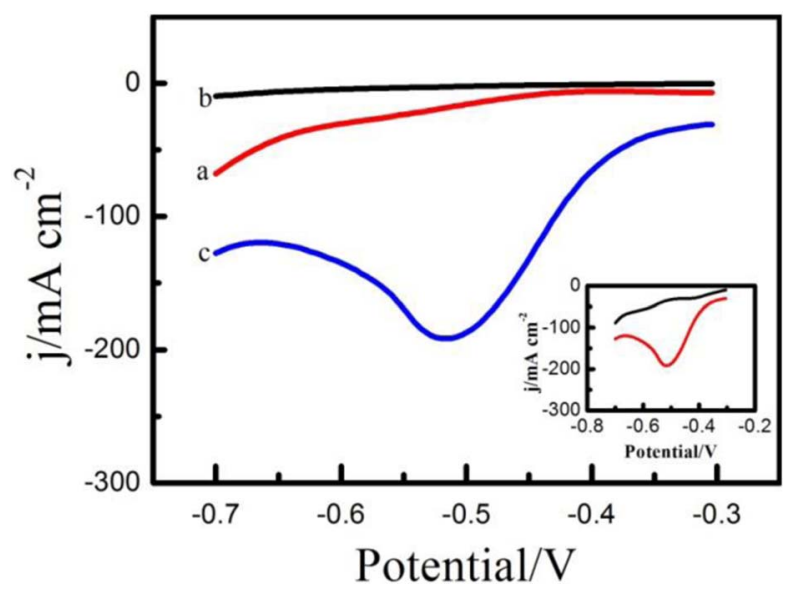

Figure 5. DPV plots of the bare MNE (a), PANI/MNE (b), and CuMSs/PANI/MNE (c) in $0.1 \mathrm{M} \mathrm{Na}_{2} \mathrm{SO}_{4}$ solution (pH 2) with $1 \mathrm{mM} \mathrm{NO} \mathrm{NO}_{3}^{-}$. Insert shows the DPV plots of CuMSs/PANI/MNE in $0.1 \mathrm{M} \mathrm{Na}_{2} \mathrm{SO}_{4}$ solution $(\mathrm{pH} 2)$ with and without $1 \mathrm{mM} \mathrm{NO}-$. 


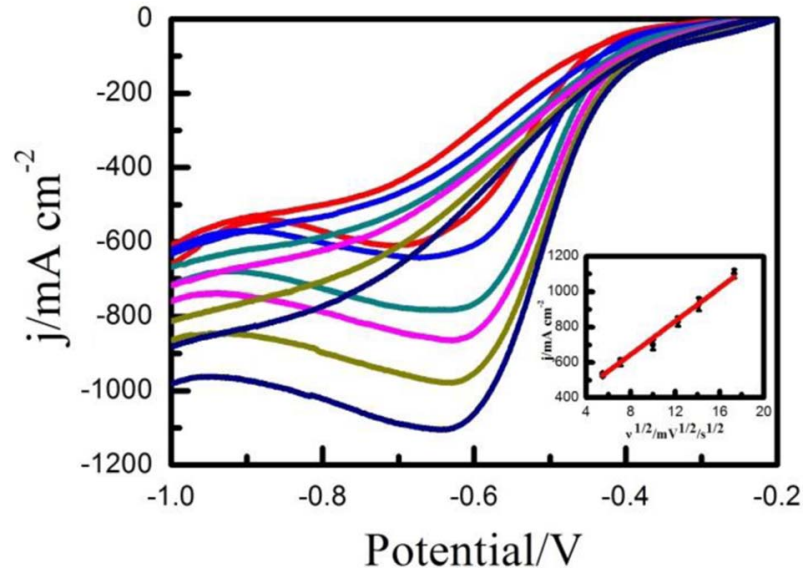

Figure 6. $\mathrm{CV}$ plots of CuMSs/PANI/MNE in $0.1 \mathrm{M} \mathrm{Na}_{2} \mathrm{SO}_{4}(\mathrm{pH}$ 2) solution containing $1 \mathrm{mM} \mathrm{NO}_{3}{ }^{-}$at different scan rates $(10-300 \mathrm{mV} / \mathrm{s})$. Insert shows the dependence of peak current density on the scan rate from $10-300 \mathrm{mV} / \mathrm{s}$.

Optimization for the response of $\mathrm{NO}_{3}^{-}$on $\mathrm{CuMSS} / \mathrm{PANI} / \mathrm{MNE}$.It should be noted that the CuMSs can be hardly deposited on the bare MNE, and be easily deposited after the modification of PANI film. Considering that the PANI film can provide much rougher surface and higher electrochemical area, the presence of PANI film may provide more binding sites for the deposition of CuMSs. This suppose was verified by ranging the deposition cycles of PANI film from 5 to 30 to investigate its influence on the deposition of CuMSs and corresponding current responses to $\mathrm{NO}_{3}{ }^{-}$. As shown in Fig. 7a, peak current density of $\mathrm{NO}_{3}^{-}$increases with the cycles from 5 to 20 , and decreases gradually after 20 cycles. With the increase of deposition from 5 to 20 cycles, more PANI could be deposited on the MNE surface and more CuMSs with excellent catalytic performance could be combined. However, when the deposition cycle is more than 20, the redundant PANI might be reunited on the electrode surface and hinder the deposition of CuMSs, which decrease the electro-caltalytic reduction performance and current response of $\mathrm{NO}_{3}^{-}$. As a result, 20 cycles was used as the optimal one for the deposition of PANI.

In this work, dc-potential was used only for the deposition of CuMSs, and our preliminary experiments indicated that the deposition potential had little effect on the ability of PANI film to provide binding sites. However, the effect of deposition time of CuMSs on the peak current density of CuMSs/PANI/MNE was investigated by ranging from 5 to $27 \mathrm{~s}$. As shown in Fig. 7b, the peak current density of $\mathrm{NO}_{3}^{-}$increases from 5 to $12 \mathrm{~s}$, and the maximum peak current density appear at the deposition time of $12 \mathrm{~s}$. And then, from 12 to 27 $\mathrm{s}$, the peak current density becomes stable. It can be concluded that with the increase of deposition time, more CuMSs will be deposited on the MNE surface which can make an enhanced performance for the electrochemical detection of $\mathrm{NO}_{3}^{-}$. After $12 \mathrm{~s}$ of the deposition time, more CuMSs will be deposited and aggregated, and the electroactive surface will not increase continually. As a result, no enhancement of peak current density will be obtained. So, in this work, the optimal deposition time was chosen as $12 \mathrm{~s}$.

The effect of $\mathrm{pH}$ of the supporting electrolyte was also investigated by ranging from $\mathrm{pH} 1$ to 5 . As shown in Fig. 7c, the peak current density of $\mathrm{NO}_{3}^{-}$increases with the $\mathrm{pH}$ from 1 to 2 , and there is the maximum current response when the $\mathrm{pH}$ is 2 . However, when the $\mathrm{pH}$ is larger than 2, the peak current density decreases gradually with the increase of $\mathrm{pH}$ from 2 to 5 . And the possible reason is that there is not enough $\mathrm{H}^{+}$to support the reduction of $\mathrm{NO}_{3}^{-}$. Thus, $\mathrm{pH} 2$ is chosen as the optimum $\mathrm{pH}$ of the electrolyte solution.

Performance of the CuMSs/PANI/MNE for $\mathrm{NO}_{3}^{-}$detection.The calibration plot for $\mathrm{NO}_{3}{ }^{-}$determination was derived from the DPV plots obtained at the CuMSs/PANI/MNE in $0.1 \mathrm{M} \mathrm{Na}_{2} \mathrm{SO}_{4}$ under the optimum conditions. As shown in Fig. 8, the peak current
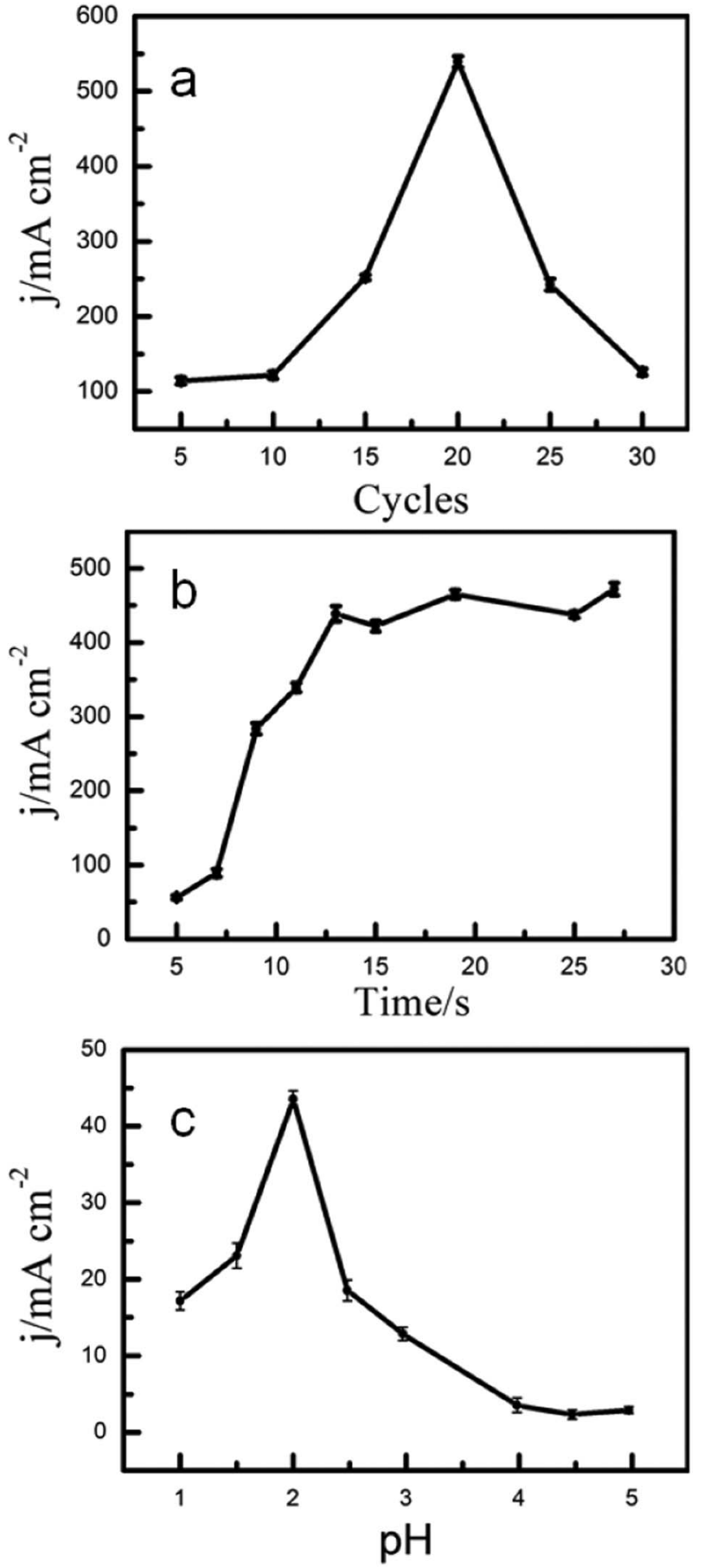

Figure 7. Effects of the deposition cycles of PANI (a), deposition time of CuMSs (b), and $\mathrm{pH}$ of electrolyte solution (c) on the peak current density.

density is linear with the concentration of $\mathrm{NO}_{3}^{-}$ranging from 0.02 to $6 \mathrm{mM}$. The equation for linear regression is $\mathrm{j}=141.69 \mathrm{C}+222.69$ $\left(\mathrm{R}^{2}=0.995\right)$ with detection limit of $8 \mu \mathrm{M}$. The comparison of the CuMSs/PANI/MNE fabricated here with other electrodes for $\mathrm{NO}_{3}^{-}$ determination is presented in Table I. It can be seen that when compared with other electrodes, the CuMSs/PANI/MNE shows superior performance for $\mathrm{NO}_{3}^{-}$determination.

Study of repeatability, interference, and stability.-The repeatability of the CuMSs/PANI/MNE was studied using the same electrode for fifty measurements and the RSD is $2.8 \%$, indicating the good repeatability of the functional MNE. To investigate the effects of possible 


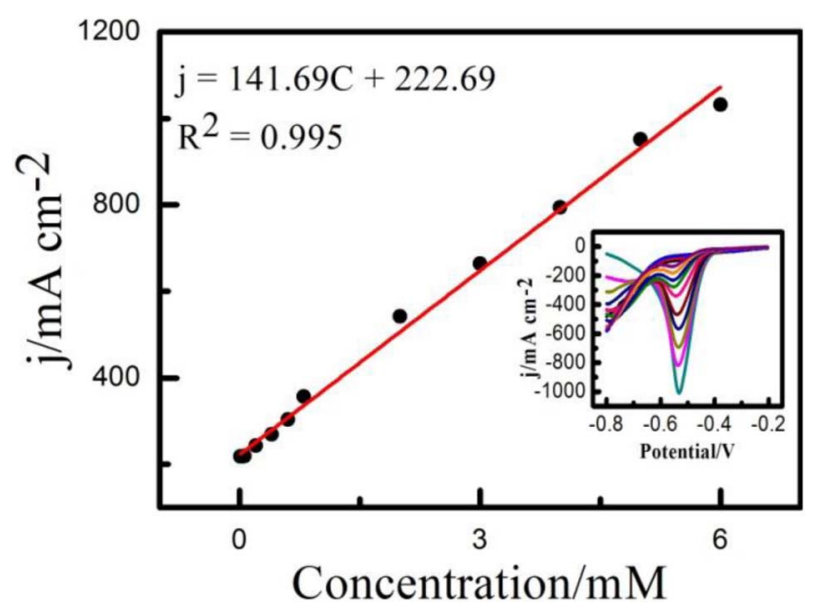

Figure 8. Calibration curve of $\mathrm{NO}_{3}{ }^{-}$at the CuMSs/PANI/MNE, where the concentrations of $\mathrm{NO}_{3}^{-}$are from 0.02 to $6 \mathrm{mM}$ in $0.1 \mathrm{M} \mathrm{Na}_{2} \mathrm{SO}_{4}(\mathrm{pH} 2)$ solution. Insert shows the voltammograms with baseline correction obtained on the CuMSs/PANI/MNE with successive addition of 0.02 to $6 \mathrm{mM} \mathrm{NO}_{3}^{-}$.

interfering species for $\mathrm{NO}_{3}^{-}$determination, the CuMSs/PANI/MNE was used in $0.1 \mathrm{M} \mathrm{Na}_{2} \mathrm{SO}_{4}$ containing $1 \mathrm{mM} \mathrm{NO}_{3}^{-}$with various foreign species added. The results show that additions of $30-$ fold $\mathrm{Cu}^{2+}$, $\mathrm{Cr}^{3+}, 10$-fold $\mathrm{Zn}^{2+}, \mathrm{As}^{3+}$, and equivalent $\mathrm{NO}_{2}^{-}$do not affect the determination of $\mathrm{NO}_{3}^{-}$. However, the addition of equivalent $\mathrm{Cl}^{-}$resulted in an enhancement of the peak current density and the shift of the peak potential towards more negative. Stability of the CuMSs/PANI/MNE was investigated by measuring the current response of $\mathrm{NO}_{3}^{-}$several times every few days and there was no obvious decrease of the peak current density after seven days. So, the CuMSs/PANI/MNE has the good repeatability, anti-interference ability, and stability.

Analytical applications.-The practical application of the functional MNE was investigated by the determination of $\mathrm{NO}_{3}^{-}$in coastal river water samples. The samples were collected on June 2018 from Yellow River Delta, DongYing, China. After collection, the samples were filtered $(0.45 \mu \mathrm{m}$ membrane filters $)$ and kept in a refrigerator at $4^{\circ} \mathrm{C}$ until detection. The concentrations of $\mathrm{NO}_{3}^{-}$in the real water samples were determined by standard addition method with the $\mathrm{CuMSs} / \mathrm{PANI} / \mathrm{MNE}$. The results obtained with the micro-needle electrode were in agreement with that detected by ion chromatography method (IC), indicating the good accuracy of the CuMSs/PANI/MNE.

\section{Table I. Comparison of the analytical performance of CuMSs/} PANI/MNE with previously reported electrodes for $\mathrm{NO}_{3}^{-}$ detection.

\begin{tabular}{ccccc}
$\begin{array}{c}\text { Modified } \\
\text { electrode }\end{array}$ & Methods & $\begin{array}{c}\text { Linear range } \\
(\mathrm{mM})\end{array}$ & $\begin{array}{c}\text { LOD } \\
(\mu \mathrm{M})\end{array}$ & Reference \\
\hline Cu electrode & DPV & $0.1-2.5$ & 11.0 & 42 \\
BiB/Cu & DPV & $0.013-3$ & 6.0 & 44 \\
Pd-Sn- microband electrode & LSV & $0.016-0.333$ & - & 45 \\
CNT-PPy-Pt electrode & CV & $0.0005-0.01$ & 0.2 & 46 \\
L-SCMNPs-CPEs & SWV & $0.1-1.982$ & 87.0 & 47 \\
Ag electrode & DPV & - & 65.0 & 48 \\
Cu electrode & Amp. & $0.1-2.5$ & 4.2 & 49 \\
CuMSs/PANI/MNE & DPV & $0.02-6$ & 8.0 & This work
\end{tabular}

CNT-PPy-Pt electrode: carbon nanotubes-polypyrrole nanocomposite platinum electrode.

L-SCMNPs-CPEs: 3,6-bis(2-[2-sulfanyl-ethylimino-methyl]-4-(4-nitrophenylazo)-phenol)pyridazine coated $\mathrm{SiO}_{2} @ \mathrm{Fe}_{3} \mathrm{O}_{4}$-carbon paste electrodes.

\begin{tabular}{|c|c|c|}
\hline Samples & $\begin{array}{l}\text { Detected by the } \\
\text { proposed method (mM) }\end{array}$ & $\begin{array}{l}\text { Detected by IC } \\
\text { method (mM) }\end{array}$ \\
\hline Sample 1 & $0.247 \pm 0.02$ & 0.231 \\
\hline Sample 2 & $0.317 \pm 0.11$ & 0.322 \\
\hline Sample 3 & $0.039 \pm 0.08$ & 0.041 \\
\hline
\end{tabular}

A comparison of results for $\mathrm{NO}_{3}^{-}$determination in coastal river water with the CuMSs/PANI/MNE and IC is shown in Table II. The results show that the CuMSs/PANI/MNE proposed here is suitable for the determination of $\mathrm{NO}_{3}^{-}$in real coastal river waters.

\section{Conclusions}

In summary, a new kind of micro-needle electrode was fabricated and functionalized with PANI film and CuMSs via electrochemical deposition method for the determination of $\mathrm{NO}_{3}^{-}$in real coastal river waters. The CuMSs/PANI/MNE was characterized by different spectroscopic and electrochemical methods. Under the optimal conditions, the CuMSs/PANI/MNE functional shows a linear response to $\mathrm{NO}_{3}^{-}$ in the concentrations ranging from 0.02 to $6 \mathrm{mM}\left(\mathrm{R}^{2}=0.995\right)$ and the detection limit is $8 \mu \mathrm{M}$. And the determination of $\mathrm{NO}_{3}^{-}$in coastal river waters was achieved by the micro-needle electrode with satisfactory results.

\section{Acknowledgments}

This work was financially supported by the Key Research and Development Plan of Yantai City (2017ZH096, 2018ZHGY076), the Key Research and Development Plan of Shandong Province (2017GHY215002).

\section{ORCID}

Haitao Han (D https://orcid.org/0000-0002-5389-6256

\section{References}

1. J. Zhang and C. Fischer, Mar. Chem., 99, 220 (2006).

2. J. Du, Y. Fa, Y. Zheng, X. Li, F. Du, and H. Yang, Chin. J. Oceanol. Limn., 32, 732 (2014).

3. M. Zhang, D. Yuan, and G. Chen, Microchim. Acta, 165, 427 (2009).

4. J. Lin and W. Chen, Am. J. Chinese. Med., 36, 635 (2008).

5. T. Yoshioka, K. Iwase, S. Nakanishi, K. Hashimoto, and K. Kamiya, J. Phys. Chem.C., 120, 15729 (2016).

6. Y. Tong and Z. He, J. Hazard. Mater., 262, 614 (2013).

7. A. Nezamzadeh-Ejhieh and Z. Nematollahi, Electrochim. Acta, 56, 8334 (2011).

8. M. Nosuhi and A. Nezamzadeh-Ejhieh, Ionics, 24, 2135 (2018).

9. M. Ghazouani, H. Akrout, S. Jomaa, S. Jellali, and L. Bousselmi, J. Electroanal. Chem., 783, 28 (2016).

10. C. Wang, L. Luo, Y. Wu, Y. Dong, Y. Chen, and J. Shi, Ionics, 19, 343 (2013).

11. M. Xin, H. Lin, J. Yang, M. Chen, X. Ma, and J. Liu, Electronal., 26, 2216 (2014).

12. X. Li, D. Wang, G. Cheng, Q. Luo, J. An, and Y. Wang, Appl. Catal. B-Environ., 81, 267 (2008).

13. Y. Wang, L. Yang, Y. Liu, Z. Mei, W. Chen, J. Li, H. Liang, A. Kuznetsov, and X. Du, Sci. Rep-Uk., 5, 10843 (2015).

14. X. Niu, Z. Wen, X. Li, W. Zhao, X. Li, Y. Huang, Q. Li, G. Li, and W. Sun, Sensor. Actuat. B-Chem., 255, 471 (2018).

15. B. Sun, F. Shan, X. Jiang, J. Ji, and F. Wang, Appl. Surf. Sci., 435, 822 (2018).

16. Y. Li, L. Tang, Y. Ning, Q. Shu, F. Liang, H. Wang, and G. Zhang, Sci. Rep-Uk, 6, 28018 (2016).

17. H. Han, W. Tao, X. Hu, X. Ding, D. Pan, C. Wang, and S. Xu, Electrochim. Acta, 289, 474 (2018).

18. J. Gamboa, R. Pen, T. Paixa, and M. Bertotti, Talanta, 80, 581 (2009)

19. S. Shariar and T. Hinoue, Anal. Sci., 26, 1173 (2010).

20. N. Aouina, H. Cachet, C. Debiemme-chouvy, and T. Tran, Electrochim. Acta, 55, $7341(2010)$.

21. Y. Li, J. Sun, C. Bian, J. Tong, and S. Xia, Chinese J. Anal. Chem., 39, 1621 (2011).

22. K. Fajerwerg, V. Ynam, B. Chaudret, V. Garç, D. Thouron, and M. Comtat, Electrochem. Commun., 12, 1439 (2010). 
23. H. Bagheri, A. Hajian, M. Rezaei, and A. Shirzadmehr, J. Hazard. Mater, 324, 762 (2017).

24. S. Sun, X. Zhang, Y. Sun, S. Yang, X. Song, and Z. Yang, ACS. Appl. Mater. Inter, 5, 4429 (2013).

25. L. Guadagnini and D. Tonelli, Sensor. Actuat. B-Chem., 188, 806 (2013).

26. M. Saito, T. Ishii, H. Fujiwara, and T. Homma, J. Electrochem. Soc., 165, E50 (2018)

27. G. Singh and S Chandra, J. Electrochem. Soc., 166, A1154 (2019).

28. W. Bai, X. Zhang, and J. Zheng, J. Electrochem. Soc.,166, H336 (2019).

29. Z. Li, Y. Shen, and Y. Li, J. Electrochem. Soc.,165, G75 (2018).

30. S. Zhang, S. Chen, Y. Zhao, J. Kang, J. Chen, B. Yan, Y. Gu, F. Yang, and Y. Cao, J. Electrochem. Soc., 166, H77 (2019).

31. R. Gurkan and N. Altunay, J. Food. Compos. Anal., 42, 141 (2015).

32. L. Bai, Y. Chen, B. Yan, Y. Chen, Z. Jing, and Y. Huang, Biomaterials, 133, 11 (2017)

33. N. Ruecha, N. Rodthongkum, D. Cate, J. Volckens, O. Chailapakul, and C. Henry, Anal. Chim. Acta, 874, 40 (2015).

34. Y. E. Miao, W. Fan, D. Chen, and T. Liu, ACS. Appl. Mater. Inter, 5, 4423 (2013).

35. D. Vonlanthen, P. Lazarev, K. See, F. Wudl, and A. Heeger, Adv. Mater, 26, 5095 (2014).

36. N. Sarkar, G. Sahoo, R. Das, G. Prusty, D. Sahu, and S. Swain, Ind. Eng. Chem. Res., 55, 2921 (2016).
37. F. Rafiqi and K. Majid, RSC. Adv., 6, 22016 (2016).

38. J. Lu, I. Do, L. Drzal, R. Worden, and I. Lee, ACS. Nano, 2, 1825 (2008).

39. Y. Zhang, J. Xiao, Y. Sun, L. Wang, X. Dong, J. Ren, W. He, and F. Xiao, Biosens. Bioelectron., 100, 453 (2018).

40. Q. Xu, H. Yuan, X. Dong, Y. Zhang, M. Asif, Z. Dong, W. He, J. Ren, Y. Sun, and F. Xiao, Biosens. Bioelectron., 107, 153 (2018).

41. Y. Zhang, J. Yin, K. Wang, P. Chen, and L. Ji, J. Appl. Polym. Sci., 128, 2971 (2013).

42. T. Paixao, J. Cardoso, and M. Bertotti, Talanta, 71, 186 (2007).

43. S. Prashanth, K. Ramesh, and J. Seetharamappa, Int. J. Electrochem., 2011, 1 (2011).

44. D. Pan, W. Lu, H. Zhang, L. Zhang, and J. Zhuang, Intern. J. Environ. Anal. Chem., 93, 935 (2013).

45. Y. Fu, C. Bian, J. Kuang, J. Wang, J. Tong, and S. Xia, Sensors, 15, 23249 (2015).

46. T. Madasamy, M. Pandiaraj, M. Balamurugan, K. Bhargava, N. Sethy, and C. Karunakaran, Biosens. Bioelectron., 52, 209 (2014).

47. A. Afkhami, T. Madrakian, H. Ghaedi, and H. Khanmohammadi, Electrochim. Acta, 66, 255 (2012).

48. J. Krista, M. Kopanica, and L. Novotny, Electroanalysis, 12, 199 (2000).

49. J. Gamboa, R. Pena, T. Paixao, and M. Bertotti, Talanta, 80, 581 (2009). 